\title{
Grammatische Mittel im Vordergrund. Pronomen, die koordinierende Konjunktion und, die Negativpartikel nicht als Vehikel ambiger Poetizität
}

\section{Poetizität als sprachliche Kategorie: Strukturalismus und neurokognitive Poetik. Zur Einleitung}

Man muss sehr viel Verstand haben, um etwas nicht zu verstehen.

Friedrich Schlegel

Der Begriff der Poetizität beruht gewöhnlich auf der Annahme, dass es Texte gibt, die sich von anderen Kunstformen und Texttypen durch besondere poetische Qualitäten und ästhetische Erkenntnismöglichkeiten unterscheiden. ${ }^{1}$ Die Debatte über die Natur der poetischen Qualität hat vor ungefähr 25 Jahrhunderten mit Aristoteles begonnen: „Denn der Geschichtsschreiber und Dichter unterscheiden sich nicht dadurch, dass sie entweder in gebundener oder in ungebundener Rede sprechen. Man könnte z.B. die Bücher Herodots ins Versmaß bringen und sie wären um nichts weniger Geschichte mit Versmaß als ohne Versmaß. Aber dadurch unterscheiden sie sich, dass der eine erzählt, was geschehen ist, der andere, wie

\footnotetext{
1 Der vorliegende Artikel basiert auf dem am 24. August 2015 beim XIII. Kongress der Internationalen Vereinigung für Germanistik (IVG) „Germanistik zwischen Tradition und Innovation” an der Tongji-Universität in Shanghai gehaltenen Vortrag „Ambiguität sprachlicher Mittel als poetische Qualität“. Eine Kurzfassung desselben ist in den Tagungsakten erschienen (Foschi 2016). Für die freundliche Aufnahme des Texts in die Breslauer Schriftenreihe Studia Linguistica bin ich dem Kollegen Lesław Cirko zu Dank verpflichtet.
} 
es hätte geschehen können; deswegen ist die Poesie auch philosophischer und ernsthafter als die Geschichte.“ (Poetik, Kap. 9, 1451a36-1452a9). Als Unterscheidungskriterium des Poetischen legt Aristoteles ein formales Prinzip fest: „der Dichter schreibt in gebundener Rede“. Nach diesem Prinzip ist Jahrhunderte lang das Poetische durch die normative Poetik als bestimmendes Merkmal der unterschiedlichen literarischen Gattungen interpretiert worden. Wie der zitierten Stelle der Poetik entnommen werden kann, gilt bei Aristoteles als weiteres Unterscheidungskriterium das Prinzip Fiktion bzw. Fiktionalität, die als eine vielmehr ästhetische, eine formale Kategorie aufzufassen ist. Es ist nämlich umstritten - die Kategorie ist u.a. von Gérard Genette (1992, 3. Kap.) in Frage gestellt worden - , dass Fiktion als eine Qualität des Texts angesehen werden kann, denn es gibt einerseits die Möglichkeit, auf literarische Weise zu erzählen, was wirklich geschehen ist (z.B. literarische Autobiographien); andererseits gibt es auch fiktionale Texte, deren literarischer Wert kontrovers ist (z.B. Trivialliteratur). Es ist das Verdienst der Strukturalisten gewesen, die Debatte auf sprachliche Ebene verschoben zu haben. Dem Formalismus-Strukturalismus verdanken wir den Begriff der Poetizität selbst (durch Aleksandr A. Potebnja, 1862) und - synonymisch dafür (vgl. Winko 2009:376) - der Literarizität (Jakobson). Die Strukturalisten fassen das Poetische als das Spezifikum der literarischen Texte auf: „dasjenige, was [ein] Werk zum literarischen Werk macht" (Jakobson 1972:31), und interpretieren es als linguistisches Phänomen, d.h. als die besondere, ,poetische“ Art der Sprachverwendung, die als das Abweichende von normaler Sprachverwendung erscheint. Mukařovský (1967:45) zufolge entsteht „poetische Benennung“ aus der Abweichung vom Bekannten, Vertrauten, von Normen und Standards, sie wird „nicht in erster Linie durch das Verhältnis zur angenommenen Wirklichkeit bestimmt, sondern durch die Art ihrer Einordnung in den Text". Das strukturalistische Vermächtnis lebt heute in mehreren Hinsichten weiter. ${ }^{2}$ Auf methodischer Ebene wird ein noch produktiver Ansatz zur linguistischen Analyse literarischer Texte durch die moderne Textstilistik gewährt, die mit erneuten textlinguistischen Kategorien und einem verfeinerten textanalytischen Instrumentarium operiert (u.a. Fix/Gardt/Knape 2008, Eroms 2008, Sandig 2006). Auf theoretischer Ebene gelten Grundauffassungen des Strukturalismus als Ausgangsprinzipien für die moderne neurokognitive Poetik (vgl. Tsur 1992). Darauf basierende Untersuchungen konnten diese durch empirische Evidenz teilweise bestätigen. Die neurokognitive Poetik (Schrott/Jacobs 2011 u.a.) versucht zu definieren, welche formalen Textelemente beim Leser besondere kognitive, emotionale und ästhetische Prozesse auslösen. Darüber hinaus versucht sie das Wie und das Warum dieser

2 Der Versuch, einen historischen Abriss der Debatte darzulegen, wäre hier kaum durchführbar. Unabdingbar für die Zwecke dieser Arbeit scheint der Hinweis auf die strukturalistische Poetik als einen nicht überholten Meilenstein für die neuere Auseinandersetzung mit dem Begriff Literarizität. Für eine ausführliche Darstellung der gegenwärtigen literaturtheoretischen Diskussion vgl. Winko (2009). 
Vorgänge auf den drei Beschreibungsebenen der Psychologie darzustellen: subjektives Empfinden, indirekt beobachtbare Hirnaktivität, objektiv-beobachtbares Verhalten. Die psychologischen Prozesse, die Gegenstand der Kognitivforschung sind, werden aufgrund von sprachlichen Textelementen unterschieden. So konnte vor Kürzerem bewiesen werden, dass es in jeglichem Text formale Elemente gibt, die beim Lesen in den Vordergrund rücken und besondere affektbetonte, „poetische“ Effekte hervorrufen (vgl. Schrott/Jacobs 2011:495). Ausgegangen wird dabei von zwei grundlegenden Prinzipien, die schon für den Strukturalismus entscheidend waren. Erstens wird Poetizität auch von der kognitiven Poetik als eine Qualität aufgefasst, die in allen Texten vorkommt, nicht nur in literarischen. Zweitens gilt auch hier als wichtige Bedingung der ästhetischen Leseerfahrung der abweichende Status der sprachlichen Zeichen. Die formale Opposition von abweichender und „normaler" Sprachverwendung wird von der kognitiven Poetik als Gegenüberstellung von Vorder- und Hintergrundelementen wahrgenommen. Als Vorder- und Hintergrundelemente werden, wie schon Miall (1977) postulierte, bestimmte Textelemente erkannt, die im Leser unterschiedliche Effekte auslösen und dabei einen „literarischen Genuss“ und eine „literarische Lesehaltung“ produzieren können. Neurokognitive Studien konnten u.a. zeigen, dass bei den Lesern ästhetische Gefühle wie Interesse, Neugier, Vergnügen und Selbstreflexion durch phonologische Phänomene, etwa Alliteration und Reim, ausgelöst werden; ferner, dass syntaktische Mittel wie Ellipsen Verständnisschwierigkeiten und Nachdenkprozesse bewirken können, und dass semantische Stilmittel wie Metapher oder Ironie das flüssige wörtliche Lesen unterbrechen, weil dabei ein übertragener Sinn gesucht werden muss (vgl. Schrott/Jacobs 2011:510-511). Textelemente, die in diesem Sinn „literarisches Lesen“ produzieren, werden als Vordergrundelemente angeführt, d.h. als Elemente, die foregrounding bewirken. Schrott/ Jacobs (2011:495) listen als Vordergrund Stilmittel, Tropen und Verfremdung auf. „Normales Lesen“ entspricht dagegen einem hochautomatisierten Verfahren der Textverarbeitung und Sinnkonstruktion, ohne Einsatz von bewussten Kontrollprozessen. Automatisches Lesen und Textverstehen wird durch die sogenannten Hintergrundelemente ermöglicht, darunter vertrautes lexikalisches Material, grammatische Normen sowie konventionelle Schemata und Situationsmodelle. Der Leser liest „,vordergründig“, wenn er die Wörter nicht nur wiedererkennt, sondern sie vielmehr interpretiert und auf ästhetische Art bewertet. Literarisches Lesen beansprucht einen abweichenden, (selbst-)reflexiven und ästhetischen Verarbeitungsprozess, der zum Nachdenken, Fühlen und Phantasieren führt (vgl. Wolf 2007). Wenn der Text nur wenig Hinweise auf Poetizität liefert, bleibt der Verarbeitungsfokus des Lesers auf der Inhaltsebene verankert. Wenn der Text dagegen genügende Vordergrundelemente enthält, rückt die sprachliche Gestaltung gegenüber dem inhaltlichen Geschehen in den Vordergrund: Jakobsons poetische Funktion der Sprache wird dabei aktiviert (vgl. Schrott/Jacobs 2011:499). Vordergrundelemente können erst dann wahrgenommen werden, wenn andere Elemente 
in den Hintergrund rücken. ${ }^{3}$ Nach dem Modell von Schrott/Jacobs (2011:495) wird der Hintergrund durch Repertoire, Vertraute Wörter, Normen und Erwartungshorizont gebildet. In diesem Sinn sind Funktionswörter und Partikeln den Hintergrundelementen zuzuordnen.

Auf diesen Thesen der kognitiven Poetik aufbauend, postuliere ich hier Folgendes: Beim literarischen Lesen werden grammatische Mittel wie Funktionswörter als vertraute Wörter normalerweise „mitgelesen“ und als Hintergrund für „abweichende"Sprachmittel wahrgenommen. Ziel des Artikels ist zu zeigen, dass grammatische Mittel dieser Art bei bestimmten Anwendungen Polysemie aufweisen und ambige Relationen ausdrücken, die auf verschiedene Weise interpretiert werden können. Es kann daher angenommen werden, dass Anwendungen dieser Art eine ,nicht-kooperative" und vielmehr poetische Funktion erfüllen. Diese kann vom Leser erkannt werden, wenn er die entsprechenden grammatischen Mittel durch eine bewusste kognitive Operation in den Vordergrund rückt. Diese These - für die ich noch keine empirischen Beweise vorlegen kann - werde ich durch linguistische Argumente untermauern. Funktionswörter wie etwa Pronomen und der koordinierende Konnektor und haben einen geringen lexikalischen Sinn, wobei sie tendenziell mehrdeutig wirken. Andere Wörter, wie z.B. die deutsche Negationspartikel nicht, haben eine deutliche lexikalische Funktion, wobei ihre jeweiligen Skopusverhältnisse nicht immer eindeutig eingeschätzt werden können. Es wird im Folgenden zuerst auf Ambiguität als paradigmatisches Element der poetischen Qualität eingegangen und auf die sprachlichen Mittel, die mehrdeutig wirken können (Kap. 2). Es soll danach (Kap. 3) an konkreten Beispielen auf textueller und syntaktischer Beschreibungsebene gezeigt werden, dass grammatische Mittel der deutschen Sprache als Vehikel der Poetizität betrachtet werden können. Meine Fallstudien betreffen Pronomen (er, es), die koordinierende Konjunktion und, schließlich die Negativpartikel nicht.

\section{Ambiguität: ein paradigmatisches Element der poetischen Qualität}

Sprachliche Ambiguität ist aus kommunikativer Sicht ein Manko, wenn man Grices Maxime der Modalität („Sei klar") Vertrauen schenkt. Sie besagt nämlich: „1. Vermeide Dunkelheit des Ausdrucks. 2. Vermeide Mehrdeutigkeit" (Grice 1979:250). Auch aus rhetorischer Perspektive ist man gemäß dem perspicuitasPrinzip genötigt, Mehrdeutigkeiten zu vermeiden. Um beim Reden verständlich zu wirken, muss man nach Cicero ,sprachrichtig reden, Worte anwenden, die gebräuchlich sind und das genau bezeichnen, was wir andeuten und erklären wollen, zweideutige Ausdrücke oder Reden vermeiden“ [,"Latine scilicet dicendo,

${ }^{3}$ Den entsprechenden Prozess hat die Gestaltpsychologie durch die Kippfiguren beschrieben (vgl. van Holt/Groeben 2005:312). 
verbis usitatis ac proprie demonstrantibus ea, quae significari ac declarari volemus, sine ambiguo verbo aut sermone" (Cicero, De oratore III, 49; Hervorhebung von M. F. A.)]. ${ }^{4}$ Allerdings kann sprachliche Ambiguität im Text dazu beitragen, bestimmte stilistische Effekte zu erreichen. Nicht zufällig ist der Mehrdeutigkeitsbegriff als aequivocatio - „die bewusste Benutzung zweideutiger Ausdrücke“ (Knape/Sieber 1998:103) - im Katalog der rhetorischen Figuren enthalten. Das Potenzial sprachlicher Mehrdeutigkeit, einen poetischen Mehrwert zu erzeugen, wird im literaturtheoretischen Umfeld unumstritten anerkannt. Die Ambiguität der Ausdrucksform wird zu den typischen Stilzügen der lyrischen Gattung oder zu den Kennzeichen der Literatur bzw. einer bestimmten literarischen Epoche gerechnet (vgl. u.a. Bode 1988). Eine Definition von Mehrdeutigkeit als Merkmal der poetischen Funktion und als Attribut der poetischen Sprache hat Jakobson 1960 geliefert: „Mehrdeutigkeit ist eine unabdingbare, unveräußerliche Folge jeder in sich selbst zentrierten Mitteilung, kurz eine Grundeigenschaft der Dichtung. [...] Der Vorrang der poetischen Funktion vor der referentiellen löscht den Gegenstandsbezug nicht aus, sondern macht ihn mehrdeutig.“ (Jakobson 1971:110f.). Die Wirkung von semantischen Ambiguitäten beim Lesen literarischer Texte, was im strukturalistischen Zeitalter als „Verfremdung“ postuliert wurde (vgl. Schwarze 1970:230), konnte die moderne Leseforschung empirisch beweisen. Durch semantisch unbestimmte Zeichen verursachte Ambiguitäten werden den Vordergrundelementen zugeordnet (vgl. Schrott/Jacobs 2011:510). Sie erzeugen „Verfremdungseffekte“, erschweren den Lesefluss und die Rekonstruktion des narrativen Handlungsstrangs oder machen diese sogar unmöglich, weil sie zu unklaren Zuordnungen der inhaltlichen Bezüge (beispielsweise zwischen den Figuren und ihren Handlungszielen oder der Kausalität der Ereignisse) führen. Aus solchen Effekten können beim Leser positive Emotionen entstehen, die gemäß den neurokognitiven Studien eine neuro-psychobiologische Basis in den mit Dopamin erzeugenden SUCH- und LUST-Systemen haben. Sie sind also mit dem menschlichen Wunsch nach Neuem und Aufregendem, der Explorationslust, verbunden (vgl. Schrott/Jacobs 2011:508). Sie können deshalb als Erzeuger von ästhetischem Genuss betrachtet werden. Festzuhalten ist: Linguistische Ambiguität kann als ästhetische Kategorie und sprachliche Ambiguität als poetische Kategorie aufgefasst werden. Im Interessenfokus liegt nun die Frage nach den formalen Sprachmitteln, die semantisch ambige Kontexte erzeugen.

In der Einleitung zum Heft 158 von „Li.Li. Zeitschrift für Literaturwissenschaft und Linguistik", die dem Thema Ambiguität gewidmet ist, weisen die Herausgeber auf die Auseinandersetzung hin, die in den 50er Jahren zwischen dem Literaturwissenschaftler Emil Staiger und dem Philosophen Martin Heidegger über die

${ }^{4}$ Aus: M. Tulli Ciceronis De oratore, in: The Latin Library. The Classics Page $<$ http://www. thelatinlibrary.com/cicero/oratore.shtml>. [Aus: Ciceros drei Bücher Vom Redner. Übersetzt und erklärt von Dr. Raphael Kühner. Stuttgart, Hoffmann. 2. Auflage. 1873, in: http://www.mediacultureonline.de]. 
Bedeutung des Worts scheint im Gedicht „Auf eine Lampe“ („Was aber schön ist, selig scheint es in ihm selbst", IV, 1) von Eduard Mörike stattgefunden hat. Es wird dabei hervorgehoben, dass die strittigen Deutungen des Worts (videtur nach Staiger, lucet nach Heidegger) und die entsprechenden Interpretationen beide möglich und berechtigt sind. Die Kontrahenten haben allerdings übersehen, dass außer scheint sämtliche Wörter im Satz (selig, es, in, ihm, selbst) mehrdeutig sind - wie fast alle lexikalischen Ausdrücke einer Sprache. Die jeweils latente Ambiguität der sprachlichen Zeichen kann sich nur im Kontext, aus der Redesituation und durch das Weltwissen der Interpreten lösen (vgl. Klein/Winkler 2010:5). Mehrdeutigkeit wird als „konstitutive Eigenschaft der menschlichen Sprache“ angesehen (Klein/Winkler 2010:6). Sie manifestiert sich auf unterschiedliche Weise, u.a. als visuelle, pragmatische, narrative Mehrdeutigkeit, als Ironie oder Witz im Sinne der Koexistenz von Antinomien (vgl. dazu Bauer/Knape et al. 2010:28ff. u. 55, Ziegler 2010:140). Der Fokus der folgenden Abschnitte liegt auf Ambiguitäten, die durch einzelne Wörter (2.1.) sowie syntaktische Strukturen (2.2.) entstehen, denen mehr als eine Interpretation zugeschrieben werden kann.

\subsection{Lexikale Mehrdeutigkeit}

Sprachliche Zeichen werden als ambig verstanden, wenn sie je nach Kontext unterschiedliche Bedeutungsvarianten aufweisen, ohne dass sie wirklich polysem sind. Es handelt sich um sprachliche Zeichen, die undeterminiert (z.B. quantitativ unbestimmte Ausdrücke wie viel, zahllos, einzeln) oder unterdeterminiert sind. Bei den ersten kann man annehmen, dass sie Vagheit ausdrücken. Unterbestimmtheit kann bei jedem Wort festgestellt werden, dessen lexikographische Definition mehr als eine Bedeutung enthält. Als Beispiele gelten Balkon, rühren und Geist. Wie Abb. 1 wiedergibt, registriert das „Digitale Wörterbuch der deutschen Sprache" der Berlin-Brandenburgischen Akademie der Wissenschaften (DWDS) für das Substantiv Balkon zwei Bedeutungen.

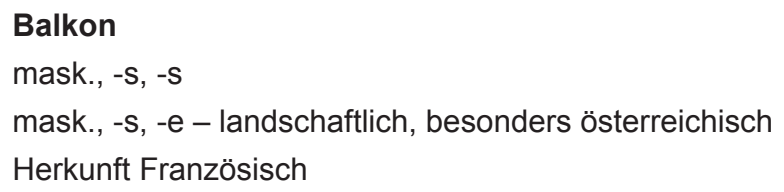

aus einem Stockwerk herausragende, mit einem Geländer versehene Plattform, auf die man vom Zimmer aus heraustritt

2

erstes emporeartiges Stockwerk im Zuschauerraum eines Theaters, Kinos

Abb. 1: Hauptbedeutungen von Balkon laut DWDS 
Für das Verb rühren gibt das DWDS sechs Bedeutungen an (Abb. 2).

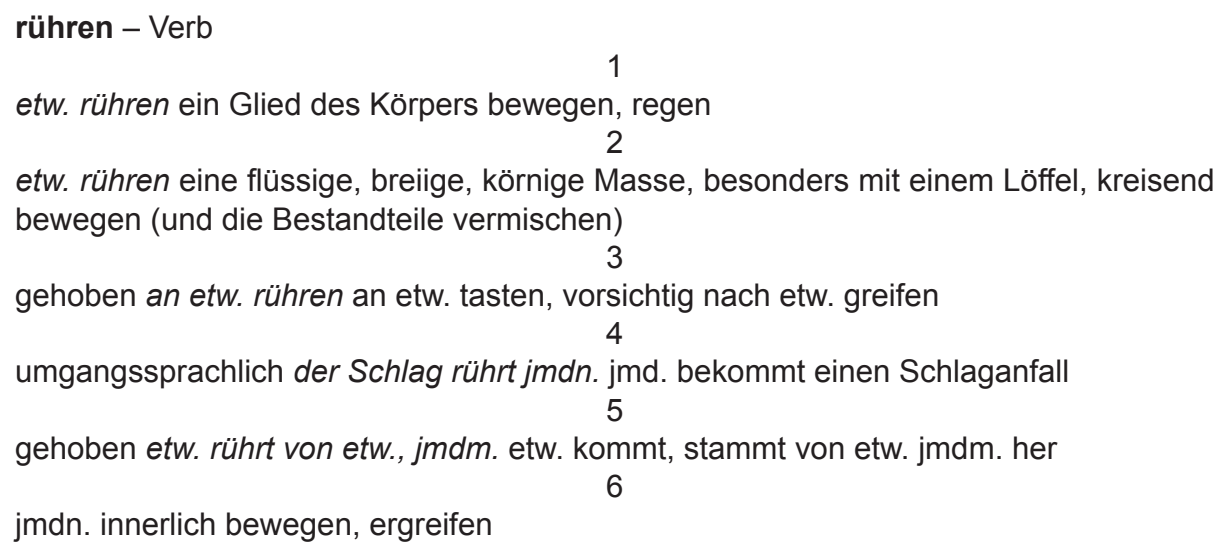

Abb. 2: Hauptbedeutungen von rühren laut DWDS

Schließlich werden im DWDS dem Substantiv Geist zwei Homographen und sechs Hauptbedeutungen zugeschrieben (Abb. 3).

Die deutsche Sprache kann die jeweilige Bedeutung unterspezifizierter Wörter durch Wortbildung genauer determinieren. Beispiele zu Balkon sind u.a. Sonnenbalkon, Balkongitter, Balkonzimmer; zu rühren: anrühren, aufrühren, glatt rühren, umrühren. In aller Regel können auch Homonyme durch den jeweiligen sprachlichen Kontext auf der Grundlage allgemeinen Weltwissens problemlos interpretiert werden. In der poetischen Sprachverwendung kann dagegen das polysemische Potential lexikalischer Einheiten offen gelassen werden, um besondere Suggestionen zu erwecken, wie z.B. die lexikalische Polysemie des Worts Geist im folgenden Gedicht von Arnfried Astel: ${ }^{5}$

(1) „Dem lebendigen Geist“

Alte Grabinschriften

In Heidelberg suchend

Fand ich diese Inschrift über dem Portal

Unserer neuen Universität.

Im Kontext wirkt das Wort Geist ersichtlich als Vordergrundelement: Der Leser stockt dabei, indem er ein widersprüchliches konzeptuelles Bild herstellt, das aus zwei verschiedenen Bedeutungsstrukturen (Nr. 1 und 6 nach DWDS, Abb. 3)

5 Sämtliche in dieser Arbeit wiedergegeben Textbeispiele aus lyrischen Texten stammen aus Conrady (2000). 


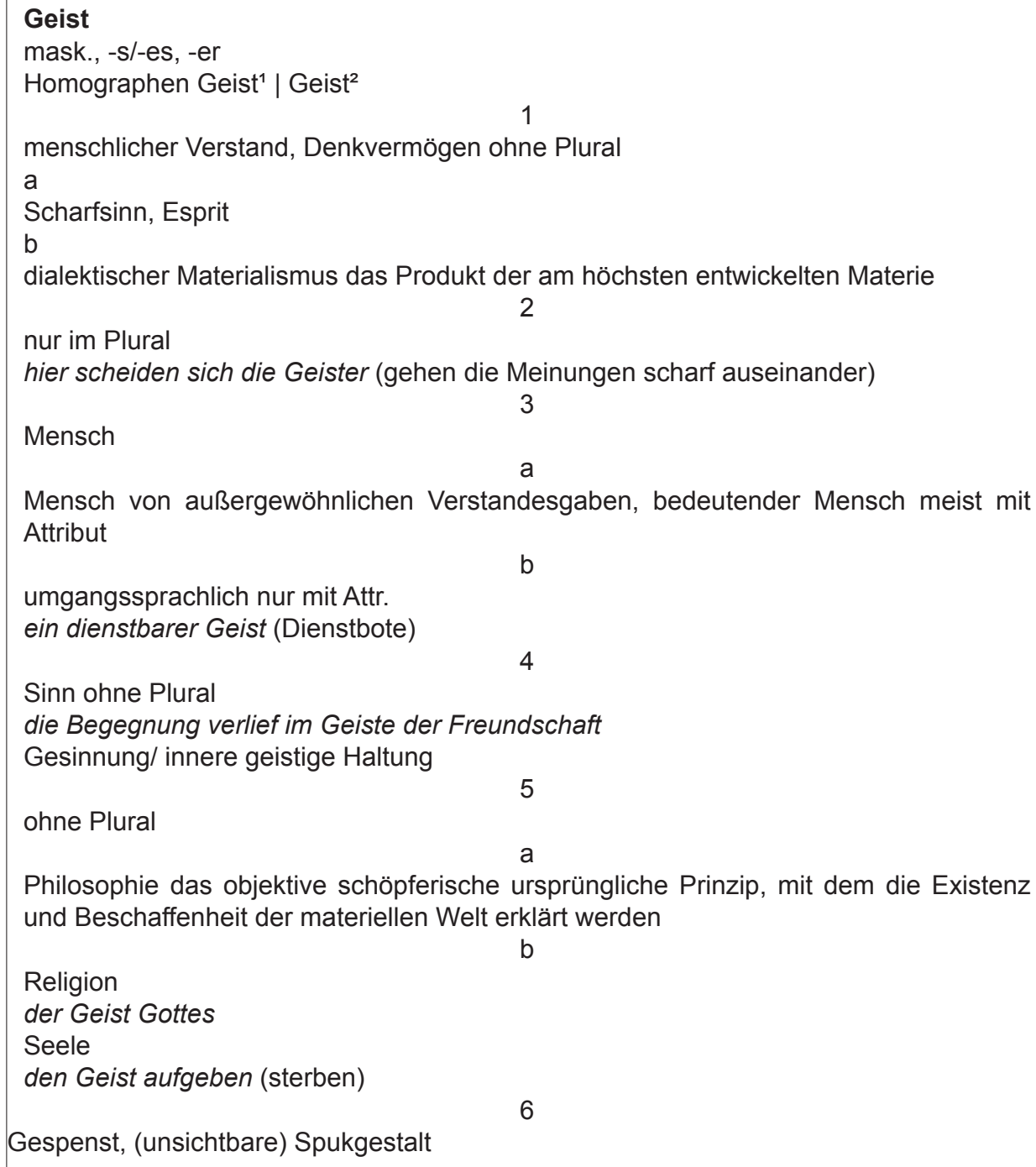

Gespenst, (unsichtbare) Spukgestalt

Abb. 3: Hauptbedeutungen von Geist laut DWDS

konstituiert ist. Lexikalische Ambiguitäten in der poetischen Sprache sind von der Literaturwissenschaft immer schon hervorgehoben und analysiert worden, vor allem mit Bezug auf Vollwörter (Substantive, Verben, Adjektive und Adverbien). So hat William Empson (1963:234) darauf hingewiesen, dass eine bestimmte Form literarischer Ambiguität durch bestimmte Wortselektionen entsteht, die als antithetische Vergleiche, Paronomasien, Wortspiele u. dgl. im Katalog der rhetorischen Figuren enthalten sind. 


\subsection{Grammatische bzw. syntaktische Mehrdeutigkeit}

Ambiguität wird durch komplexe sprachliche Zeichen und syntaktische Strukturen ausgedrückt, die mehr als nur eine Interpretation zulassen (vgl. dazu WinterFroemel/Zirker 2010:77). Mehrdeutigkeit kann sich auf unterschiedlichen syntaktischen Ebenen ergeben, wie die folgenden Beispiele zeigen:

(1) Es ist ein Zischelwind, der leere Hütten umkreist - (Georg Trakl, De profundis, I, 3)

(2) Sein Blick ist vom Vorübergehn der Stäbe / so mild geworden, daß er nichts mehr hält. (Rainer M. Rilke, Der Panther, I, 1-2)

(3) Die Erde krallt die Hand (August Stramm, Gefallen, I, 2)

Das im Beispiel (1) enthaltene Kompositum Zischelwind stellt kein lexikalisiertes Wortbildungsprodukt dar. Im DWDS wird kein Eintrag dafür registriert, und es werden keine Komposita mit zischel(n) angegeben. Eine Internetsuche führt zu wenigen Ergebnissen dieser Art, d.h. Komposita wie Zischelmund, Zischelstimme, die ausnahmsweise in literarischen Werken vorkommen. Wind als Bezeichnung für eine „spürbare stärkere Luftbewegung“ und das Verb zischeln als Ausdruck der Handlung: ,(in ärgerlichem oder eindringlich scharfem Ton) in einer Sprechweise flüstern, bei der besonders die Zischlaute hervortreten" (oder: abwertend versteckt und flüsternd Böses über jmdn., etw. reden, klatschen) (vgl. DWDS) stellen eine ungewöhnliche semantische Kombination dar, die reichhaltige Assoziationen wecken kann. Beispielweise denkt Kleefeld (1985:246) dabei an ein gefährliches Tier, ein aggressives Raubtier, etwa an einen Wolf (wobei das Bild nicht aus dem Kompositum allein, sondern aus der ganzen Wortgruppe ein Zischelwind entsteht mit ihrem durch den Relativsatz der leere Hütten umkreist ausgedrückten Attribut), aber auch an eine (zischelnde) Schlange. Da die Schlange eine phallische Bedeutung hat, versteht schließlich der Interpret Zischelwind als ,ein Bruder des Blaubart, als ein phallischer Aggressor" (Kleefeld 1985:247). In (2) wird Ambiguität durch die Nominalgruppe (das) Vorübergehn mit dem Genitivattribut der Stäbe erzeugt. Dabei kann die Genitivergänzung des nominalisierten Verbs vorübergehen als genitivus subjectivus bzw. als genitivus objectivus gedeutet werden. Im ersten Fall versteht man dann, dass die Stäbe am Panther (seinem Blick) vorübergehen. Im zweiten Fall, dass der Panther an den Stäben vorübergeht, d.h. sie ignoriert. Beispiel (3) zeigt einen Satz, der eine doppelte Deutung der syntaktischen Funktionen der Verbergänzungen zulässt. Die beiden Konstituenten die Erde und die Hand können als Subjekt bzw. Objekt oder auch umgekehrt ausgelegt werden.

Durch syntaktische Strukturen entstandene Ambiguitäten ergeben sich im Alltagsgespräch seltener als in der Schriftsprache. Manche Ausdrücke sind gesprochen eindeutig und nur schriftlich mehrdeutig (vgl. Lang 2010). Mehrdeutige Strukturen können nämlich durch die Prosodie desambiguiert werden, wie Bei- 
spiel (4) durch seine Varianten (4a) und (4b) zeigt (vgl. dazu Bauer/Knape et al. 2010:45): ${ }^{6}$

(4) Barbara isst nur Feigen aus Tunesien.

(4a) Barbara isst nur FEIGEN aus Tunesien. (= keine Datteln oder Granatäpfel aus Tunesien).

(4b) Barbara isst nur Feigen aus TUNESIEN (= keine Feigen aus Marokko oder Sizilien). ${ }^{7}$

Auf Grund ihres seltenen Vorkommens in der Alltagskommunikation werden syntaktische Kombinationen, die Ambiguität erzeugen, als „abweichend“ wahrgenommen. Baumgärtner (1965:75) bezeichnet seltene syntaktische Strukturen und ungewöhnliche Verwendungen als ,grammatische Mehrdeutigkeit“ und berücksichtigt sie als typische Elemente der poetischen Sprache. In seinen Untersuchungen zur Dichtungssprache von Dante Alighieri hat Schwarze (1970) eine Unterscheidung zwischen syntaktischen Strukturen, die rhetorische Zwecke verfolgen, und ,authentischen“ Ambiguitäten vorgenommen. Dabei spricht er von Strukturverschleierung und Strukturhomonymie (Schwarze 1970:228f.). Im ersten Fall geht es um schwer zugängliche syntaktische Strukturen, deren textueller Sinn nicht offen, sondern präzise gemeint ist. Als „verschleierte“ Strukturen sind sie charakteristisch für den Stil, den Dante in seiner poetologischen Schrift „De vulgari eloquentia“ (II, vi, 6) als ,gradus constructionis excellentissimus“ bezeichnet (,et sapidus et venustus etiam et excelsus“) (Schwarze 1970:251).

\section{3. „Ambige“ Mittel der deutschen Sprache}

Die meisten Analysen über die poetische Sprache und ihre Ambiguität beruhen auf literarischen Texten. Dort werden abweichende Verwendungen von bestimmten grammatischen Mitteln beobachtet. Die Klassifikation der poetischen Sprachmittel entsteht aus literarischen Texten. So wird Poetizität - als Sammelbegriff für die sprachlichen Mittel, die poetische Texte charakterisieren - tautologisch bestimmt. Ich versuche, systematisch und a priori vorzugehen, um Poetizität in diesem Sinn auf der Grundlage der grammatischen Beschreibung der deutschen Sprache zu isolieren. Mit anderen Worten: Bestimmte grammatische Mittel hervorzuheben, aus denen mehrdeutige Konstruktionen entstehen können. Erst danach wird ihre „poetische“ Anwendung in literarischen Texten überprüft. Meine

${ }^{6}$ In (4a) - (4b) zeigt Großschreibung das jeweilige Wort im Satz an, dessen Bedeutung für das Informationsprofil zentral ist und prosodisch hervorgehoben werden muss.

7 Bzw. (je nach Projektion des Fokus) (vgl. Uhmann 1991:100) ,keine Datteln oder Granatäpfel aus Marokko oder Sizilien'. Bei (4b) bleibt also eine Mehrdeutigkeit, die nur in der Situation und aus dem pragmatischen Kontext gelöst werden kann. 
Beispiele betreffen Pronomen (er, es) (vgl. dazu Foschi 2012, 2010, 2009), die koordinierende Konjunktion und sowie die Negationspartikel nicht (Foschi 2015).

\subsection{Pronomen}

Pronomen sind semantisch undeterminierte Zeichen, die durch formale Referenz bestimmt werden. Um kooperativ zu wirken, müssen Texte formale Beziehungen makellos realisieren und definite Referenz ausdrücken. Wenn dies nicht der Fall ist, realisiert sich im Text die poetische Qualität der Pronomen, der ihre latente Mehrdeutigkeit entspricht. Die Ambiguität der Pronomen wirkt besonders stark, wenn mehrere Referenzketten vorhanden sind, vor allem aber, wenn es sich um homonyme Ketten handelt, d.h. genus- und numerus-identische Pronomen, die auf unterschiedliche Worte bzw. Wortgruppen hinweisen. In dieser Sichtweise beobachten wir im folgenden Beispiel - aus Kafkas Erzählung Eine kaiserliche Botschaft - eine Referenzkette, die zwei Maskulina Singular enthält: der Kaiser und der Bote. Die sprachlichen Elemente, die uns interessieren, sind fett hervorgehoben und durchnummeriert:

(5) Der Kaiser ${ }^{1}$ - so heißt es - hat Dir, dem Einzelnen, dem jämmerlichen Untertanen, dem winzig vor der kaiserlichen Sonne in die fernste Ferne geflüchteten Schatten, gerade Dir hat der Kaiser ${ }^{2}$ von seinem ${ }^{3}$ Sterbebett aus eine Botschaft gesendet. Den Boten ${ }^{4}$ hat $\mathbf{e r}^{5}$ beim Bett niederknien lassen und $\mathbf{i h m}^{6}$ die Botschaft ins Ohr geflüstert; so sehr war ihm ${ }^{7}$ an ihr gelegen, daß er ${ }^{8}$ sich sie noch ins Ohr wiedersagen ließ. Durch Kopfnikken hat $\mathbf{e r}^{9}$ die Richtigkeit des Gesagten bestätigt. Und vor der ganzen Zuschauerschaft seines ${ }^{10}$ Todes - alle hindernden Wände werden niedergebrochen und auf den weit und hoch sich schwingenden Freitreppen stehen im Ring die Großen des Reichs - vor allen diesen hat er ${ }^{11}$ den Boten ${ }^{12}$ abgefertigt. Der Bote ${ }^{13}$ hat sich gleich auf den Weg gemacht; ein kräftiger, ein unermüdlicher Mann ${ }^{14}$; einmal diesen, einmal den andern Arm vorstreckend schafft er $^{15}$ sich Bahn durch die Menge; findet er $^{16}$ Widerstand, zeigt er $^{17}$ auf die Brust, wo das Zeichen der Sonne ist; er ${ }^{18}$ kommt auch leicht vorwärts, wie kein anderer. [...] (Kafka 1994:221).

Die Abfolge von 5 bis 11 besteht aus identischen er-Formen, die für zwei verschiedene Referenten stehen. Renominalisierungen werden da realisiert, wo es eigentlich aus kommunikativer Sicht unnötig wäre: bei den Pronomen 1-2 und 12-13. Die Abfolge der er-Formen 13-14, die sich vor dem er-Pronomen 15 realisiert, stellt zuerst ein determiniertes, danach ein undeterminiertes Nomen dar. Die Pronominalreferenz in Kafkas Text zeigt sich als leicht verfremdend. Vor allem bei den er-Formen 7 und 9 muss der Leser zweimal überlegen, ob vom Kai- 
ser oder vom Boten die Rede ist. Textstellen mit homonymen Pronominalketten ermöglichen mehrere Interpretationen der semantischen Referenz. Interessante Ambiguitäten können vor allem dadurch entstehen, dass es-Pronominalformen mit unterschiedlichen Funktionen in engen Kontexten verwendet werden. Als Beispiel dafür gilt eine Textstelle aus Kafkas „Vor dem Gesetz“. Vorhandene es-Pronomen sind durchnummeriert. Die Wortgruppen, die als Kandidaten der pronominalen Referenz zu berücksichtigen sind, werden durch eckige Klammern isoliert.

(6) Aber der Türhüter sagt, daß er ihm jetzt den Eintritt nicht gewähren könne. Der Mann überlegt und fragt dann, [ob er also später werde eintreten dürfen]. «Es ${ }^{1}$ ist möglich», sagt der Türhüter, «jetzt aber nicht.» Da das Tor zum Gesetz offensteht wie immer und der Türhüter beiseite tritt, bückt sich der Mann, um [durch das Tor in das Innere zu sehn]. Als der Türhüter das $^{2}$ merkt, lacht er und sagt: «Wenn $\mathbf{e s}^{3}$ [was?] dich so lockt, versuche $\mathbf{e s}^{4}$ doch, trotz meines Verbotes hineinzugehn. [...] (Kafka 1994:211).

Im Text sagt der Türhüter: „Es ist möglich“. Vermutlich weist er dabei durch das phorische $e s^{1}$ auf den Inhalt der Frage hin, die sein Gesprächspartner gestellt hat, nämlich: „ob er also später werde eintreten dürfen“. Als Referent des phorischen Pronomens $\left(d a s^{2}\right)$ versteht man hier die Angelegenheit, die der Türhüter erwähnt - dass der Mann sich bückt, „um durch das Tor in das Innere zu sehn”. Später im Text erscheinen zwei homographe es-Formen, deren Referenz bzw. Leere nicht ganz klar zu bestimmen ist, weil $d a s^{2}$ anaphorische Erwartungen bezüglich $e s^{3}$ und $e s^{4}$ öffnet. So kann sich entweder der Sinn ergeben, der aus der linearen Lektüre entsteht (vgl. Wenn es dich so lockt, durch das Tor in das Innere zu sehn, versuche es), oder ein anderer Sinn, der aus einem kataphorischen Verweis von $e s^{3}$ und $e s^{4}$ entstehen würde (vgl. Wenn es dich so lockt, trotz meines Verbots hineinzugehen, versuche es).

\subsection{Die koordinierende Konjunktion und}

Die durch und ausgedrückten Relationen entsprechen nicht immer dem Normalfall der additiven Verknüpfung. Im „Digitalen Wörterbuch der deutschen Sprache" werden der verknüpfenden Funktion von und auf syntagmatischer Ebene fünf semantische Hauptfunktionen zugeschrieben: 1. als Ausdruck der Aufzählung; 2. als Bezeichnung von Verstärkung, Steigerung, Intensivierung; 3. als Verbindung mehrerer Merkmale bei einer Person oder Sache bzw. Gegensätzliches umfassend; 4. als Ausdruck der Beziehung einer Person zu einer Sache / zu einer weiteren Person; 5. als Ausdruck der Addierung. Auf syntaktischer Ebene drückt und unterschiedliche Relationen aus, u.a. Konzessivität, Konditionalität, Temporalität, Konsekutivität, Adversativität. Das poetische Potenzial der koordinieren- 
den Konjunktion und liegt also an seiner Unterbestimmtheit (Blühdorn 2010:11). Als Zeichen seiner Wirkung als grammatisches Mittel der Poetizität kann zuerst einmal gelten, dass und in lyrischen Texten häufig vorkommt, in einigen davon mit ersichtlich hoher Frequenz. Das ist z.B. bei Hofmannsthals Ballade des äußeren Lebens (1895) der Fall, wo und 25-mal erscheint, davon 11-mal an Verseingängen. Die und-Vorkommen im wiedergegebenen Text des Gedichts (7) sind fett hervorgehoben und durchnummeriert.

(7) Und ${ }^{1}$ Kinder wachsen auf mit tiefen Augen, die von nichts wissen, wachsen auf und ${ }^{2}$ sterben, Und $^{3}$ alle Menschen gehen ihre Wege.

Und $^{4}$ süße Früchte werden aus den herben Und $^{5}$ fallen nachts wie tote Vögel nieder Und $^{6}$ liegen wenig Tage und ${ }^{7}$ verderben.

Und $^{8}$ immer weht der Wind, und ${ }^{9}$ immer wieder Vernehmen wir und ${ }^{10}$ reden viele Worte Und $^{11}$ spüren Lust und ${ }^{12}$ Müdigkeit der Glieder.

Und $^{13}$ Straßen laufen durch das Gras, und ${ }^{14}$ Orte Sind da und ${ }^{15}$ dort, voll Fackeln, Bäumen, Teichen, Und $^{16}$ drohende, und ${ }^{17}$ totenhaft verdorrte ...

Wozu sind diese aufgebaut? Und ${ }^{18}$ gleichen Einander nie? Und ${ }^{19}$ sind unzählig viele? Was wechselt Lachen, Weinen und ${ }^{20}$ Erbleichen?

Was frommt das alles uns und ${ }^{21}$ diese Spiele, Die wir doch groß und ${ }^{22}$ ewig einsam sind Und $^{23}$ wandernd nimmer suchen irgend Ziele?

Was frommt's, dergleichen viel gesehen haben? Und $^{24}$ dennoch sagt der viel, der ,Abend sagt, Ein Wort, daraus Tiefsinn und ${ }^{25}$ Trauer rinnt

Wie schwerer Honig aus den hohlen Waben.

Die wiederholte Reihung durch die Konjunktion und in den Verseingängen der Ballade dient nach den Literaturwissenschaftlern Leiß/Stadler (1997:274) dazu, „eine einfache Addition der erfassten Erscheinungen“ zu konstituieren. Van Rinsum/van Rinsum (1986:56) heben dagegen hervor, dass die im Gedicht durch und 
ausgedrückten Relationen nicht immer dem Normalfall der additiven Verknüpfung entsprechen. Dabei wird die Konjunktion und im Gedichttext als Mittel, Temporalität auszudrücken, genauer als „Glied der sinnlosen Kette Zeit“ interpretiert. Bei näherer Betrachtung fällt zuerst einmal auf, dass nicht sämtliche Vorkommen von und im Gedicht syntaktisch gleich sind. Es gibt vielmehr vier und-Typologien:

(a) und-Konnektoren 12, 15, 16-17, 20, 21, 22, 25 verbinden Wörter bzw. Wortgruppen zu Satzgliedern;

(b) und-Konnektoren 2, 5-7, 10-11, 18-19, 23 verbinden Sätze zu komplexen Sätzen. Die Sätze haben dasselbe Subjekt (im Text unterstrichen);

(c) und-Konnektoren 3, 4, 8, 9, 13, 14, 24 verbinden Sätze, die unterschiedliche Subjekte haben;

(d) und-Konnektor 1 scheint keine Rolle als Konnektor zu haben, da es keinen vorhergehenden Satz gibt, der als Konjunkt des Satzes Kinder wachsen auf... gelten kann.

In den verschiedenen Fällen übernimmt $u n d$ unterschiedlich interpretierbare semantische Rollen. Welcher Art diese sind, kann bei den Konnektoren der (a)Typologie wie folgt gedeutet werden:

- und-Konnektor 12 umfasst Gegensätzliches (Lust, Müdigkeit der Glieder);

- und-Konnektor 15 verbindet ein alliterierendes Wortpaar ( $d a, d o r t)$;

- und-Konnektoren 16-17 vereinigen Merkmale (voll Fackeln, Bäumen, Teichen / drohende, totenhaft verdorrt) bei einer Sache (Orte);

- und-Konnektor 20 umfasst Gegensätzliches (Lachen, Weinen, Erbleichen);

- und-Konnektor 21 drückt die Beziehung einer Person (wir) zu einer Sache (diese Spiele) aus;

- und-Konnektor 22 vereinigt zwei Merkmale (groß, ewig einsam) bei einer Person (wir);

- und-Konnektor 25 verbindet ein alliterierendes Wortpaar (Tiefsinn, Trauer).

Die jeweilige ausgedrückte Relation bei den und-Konnektoren der (b)-Typologie kann nicht genau festgelegt, aber vielmehr bei den Konnektoren 2, 5-7, 10 11 als temporal oder konsekutiv interpretiert werden. Bei den und-Konnektoren 18-19 und bei dem Konnektor 23 ist sie als mögliche additive bzw. adversative Relation zu deuten, in beiden Fällen allerdings mit einer gewissen Unsicherheit. Noch unbestimmter wirkt die Relation bei den (c)-und-Konnektoren. Die entsprechenden Konjunkte mit unterschiedlichen Subjekten wirken als lockerer verbunden, auch weil sie durch Interpunktionszeichen getrennt sind. Die Funktion von und scheint in diesen Fällen eine vorwiegend pragmatische zu sein: und erstellt eine „künstliche“ Verbindung zwischen Textteilen - die und-Aussage und das Vorhergesagte. Das ist ganz besonders beim ersten und im Gedichttext 
der Fall, wo es kein Vorhergesagtes gibt - bis auf den Titel des Gedichts mit seiner einleitenden Funktion.

\subsection{Die Negationspartikel nicht}

Auch der Gebrauch der Negationspartikel nicht kann zu semantischen Ambiguitäten führen. Die semantische Funktion der Negationspartikel ist anschaulich, nämlich, Äußerungen zu negieren. Ambiguitäten können dennoch entstehen in Bezug auf zweierlei: 1. die Bestimmung dessen, was negiert wird; 2. die Kontrastierung dessen, was statt der negierten Äußerung als geltend gemacht werden soll. Der Bezugsbereich bzw. der Skopus der Negationspartikel ist stets der gesamte Satz, wie im folgenden Beispiel:

(8) Dein geborgtes Sonnenlicht / soll mich nicht blenden (Willy Bartock, Niederrhein I, 3-4).

Dabei kann die Negationspartikel einen speziellen Bezugsausdruck (d.h. einen Fokus) haben. Der Negationsfokus wird im Satz durch die Position des nicht (links vom negierten Ausdruck) und/oder durch den Akzent hervorgehobenen, wie die unterschiedlichen Markierungen in den Textvarianten $(8 a)-(8 c)$ zeigen:

(8a) Dein geborgtes Sonnenlicht / soll mich nicht blenden.

(8b) Dein geborgtes Sonnenlicht / soll nicht mich blenden.

(8c) Nicht dein geborgtes Sonnenlicht / soll mich blenden.

In der Schriftsprache können unscharfe Fokussierungen dann entstehen, wenn die Position des nicht zwischen zwei Satzgliedern ,schwankt", wie z.B. in (9). Je nachdem, wie beim Lesen Pausen und Akzente gesetzt werden, fällt der Fokus von nicht auf ein anderes Satzglied. Vgl. Zeilen 1-2: Noch bin ich nicht angekommen / bei euch [Pause] nicht bei mir; Noch bin ich nicht angekommen / bei euch nicht [Pause] bei mir.

(9) Noch bin ich nicht angekommen

bei euch nicht bei mir

bei uns nicht bei dir

am Tag nicht im Traum

im Ton nicht im Baum

mit Rad nicht mit Bahn [...] (Michael Wüstefeld, Kleines Rondeau)

Da die Schriftsprache den prosodischen Akzent nicht erkennen lässt, können Ambiguitäten dadurch entstehen, dass Satzglieder als Fokus der Negation nicht eindeutig isoliert werden können, wie in den Beispielen (10)-(12). In (10) kann als Fokus 
entweder enden oder enden gerecht interpretiert werden; in (11) über die Mauer oder über die Mauer zum Nachbarn; in (12) der Gruß sowie der Gruß aus dem Nichts.

(10) Zeichen, Farben, es ist ein Spiel, ich bin bedenklich, es möchte nicht enden gerecht. (Johannes Bobrowski, Immer zu benennen, II, 1-4)

(11) Unser Atem hebt sich nicht über die Mauer zum Nachbarn. (Olly Komenda-Soentgerath, Individuum, I, 7-10)

(12) Geschrieben wird nicht Der Gruß aus dem Nichts: (Heinz Czechowski, Flußfahrt, 2, 4-5)

Im Allgemeinen kann die Verwendung der Negation als „poetisch“ angesehen werden. Nach optimaler Praxis vom kooperativen Sprechhandeln (vgl. Deppermann/Blühdorn 2013) müsste bei der Negation nicht nur das genannt werden, was nicht gelten, sondern auch kontrastierend das, was geltend gemacht werden soll, nach dem Modell der „kooperativen Negation“ in den folgenden ausgeweiteten Varianten (8i) - (8iv) vom Beispiel (8):

(8i) Dein geborgtes Sonnenlicht / soll mich nicht blenden. Ich will gut sehen können.

(8ii) Dein geborgtes Sonnenlicht / soll mich nicht blenden, sondern erwärmen.

(8iii) Dein geborgtes Sonnenlicht / soll nicht mich blenden. Es soll eine andere Person blenden.

(8iv) Nicht dein geborgtes Sonnenlicht / soll mich blenden. Das Neonlicht soll mich blenden.

Wenn keine explizite Kontrastierung vorhanden ist, entsteht durch Negation eine offene Interpretation dessen, was gelten soll. In diesem Fall wirkt die Negation nicht kooperativ, sondern ,poetisch“. Beispiele dieser Art sind (8) und $(8 \mathrm{a})-(8 \mathrm{c})$, im Folgenden als (8.01) - (8.04) wiedergegeben. Dabei ist die Interpretation bei Satznegation (8.01) völlig offen. Bei fokussierten Negationen $(8.02-8.04)$ wird die Interpretation teilweise gesteuert (vgl. Blühdorn 2012:294f.), sie bleibt auf jeden Fall offen. Dadurch, dass die Konstruktion autonome Bilder von Seiten der Leser ermöglicht, kann Negation als poetisches Mittel an sich angesehen werden. 
(8.01) Dein geborgtes Sonnenlicht / soll mich nicht blenden. (Was soll geschehen?)

(8.02) Dein geborgtes Sonnenlicht / soll mich nicht blenden. (Was soll es tun?)

(8.03) Dein geborgtes Sonnenlicht / soll nicht mich blenden. (Wen soll es blenden?)

(8.04) Nicht dein geborgtes Sonnenlicht / soll mich blenden. (Welches Agens soll daslyrische Ichblenden?)

Explizite Kontrastierung kommt in lyrischen Texten nicht häufig vor. Eine Ausnahme scheinen in dieser Hinsicht Brechts Texte darzustellen, wo mehrere Beispiele dieser Art vorzufinden sind (13)-(15). Brechts Verwendung ,nicht poetischer" Negation kann als Kennzeichnen seines besonderen poetischen Stils angesehen werden.

(13) Die grünen Boote und die lustigen Segel des Sundes

Sehe ich nicht. Von allem

Sehe ich nur der Fischer rissiges Garnnetz. (Bertolt Brecht, Schlechte Zeit für Lyrik, III, 1-3)

(14) Seine Wünsche nicht erfüllen, sondern vergessen

Gilt für weise. (Bertolt Brecht, An die Nachgeborenen I, V, 7 -8)

(14) $\mathrm{Daß}$ die Völker nicht erbleichen

Wie vor einer Räuberin

Sondern ihre Hände reichen

Uns wie andern Völkern hin. (Bertolt Brecht, Kinderhymne II, 1 -4)

\section{Fazit}

Pronominale Referenz sowie Funktionswörter im Allgemeinen und weitere „normkonforme“ Sprachmittel wie die Negation (Schrott/Jacobs 2011:510, mit spezifischem Bezug auf Pronomen) werden von der kognitiven Poetik tendenziell als Hintergrundelemente verstanden, die Hinweisreize auf mikrostruktureller Ebene produzieren. Die Erkennung bestimmter grammatischer Mittel erfolge daher automatisch und bringe zum Verstehen inhaltsbezogener Relationen. Diese Arbeit hat versucht zu zeigen, dass unterdeterminierte grammatische Zeichen referenzambige Strukturen schaffen können. Wenn diese bewusst in den Vordergrund der Aufmerksamkeit gerückt werden, können sie „literarische“ Reaktionen bewirken, mit interessanten Konsequenzen für den persönlichen Genuss von Literatur, für hermeneutische Zwecke und für die Praxis der literarischen Übersetzung. 


\section{Literaturverzeichnis}

BAUER Matthias / KNAPE Joachim / Koch Peter / WINKLER Susanne, 2010, Dimensionen der Ambiguität, in: LiLi. Zeitschrift für Literaturwissenschaft und Linguistik 40, 158 [Ambiguität. Hrsg. von Klein W./Winkler S.], S. 7-75.

BAUMGÄRTNER Klaus, 1965, Formale Erklärung poetischer Texte, in: Kreuzer H./Gunzenhäuser R. (Hrsg.), Mathematik und Dichtung. Versuche zur Frage einer exakten Literaturwissenschaft, München, S. 67-84.

BLÜHDORN Hardarik, 2012, Negation im Deutschen. Syntax, Informationsstruktur, Semantik, Tübingen.

BodE Christoph, 1988, Ästhetik der Ambiguität: Zu Funktion und Bedeutung von Mehrdeutigkeit in der Literatur der Moderne, Tübingen.

Conrady = Der Neue Conrady. Das große deutsche Gedichtbuch. Von den Anfängen bis zur Gegenwart. Hrsg. von Conrady Karl Otto, 2000, erweiterte und aktualisierte Neuausgabe, Düsseldorf/ Zürich.

DEPPERMANN Arnulf / BLÜHDORN Hardarik, 2013, Negation als Verfahren des Adressatenzuschnitts: Verstehenssteuerung durch Interpretationsrestriktionen, in: Deutsche Sprache. Zeitschrift für Theorie Praxis Dokumentation 41/1, S. 6-30.

DWDS = Das Digitale Wörterbuch der deutschen Sprache der Berlin-Brandenburgischen Akademie der Wissenschaften, in: <http://www.dwds.de>.

EMPSON William, 1963, Seven Types Of Ambiguity, London.

EROMS Hans-Werner, 2008, Stil und Stilistik. Eine Einführung, Berlin.

FIX Ulla / GARDT Andreas / KNAPE Joachim (Hrsg.), 2008, Rhetorik und Stilistik. Ein internationales Handbuch, Berlin/New York.

Foschi AlBerT Marina, 2009, Pronomi ambigui in Kafka, in: Carmassi C./Cermelli G./ Foschi Albert M./Hepp M. (Hrsg.), Wo bleibt das „Konzept”? Festschrift für Enrico De Angelis, München, S. 218-236.

FosCHI AlBERT Marina, 2010, Pronominalizzazione e comunicazione scientifica: lo es in Freud, in: SliFo - Studi linguistici e filologici online 8/2, S. 361-382, in: <http://www.humnet.unipi.it/ slifo/>.

FosCHI ALBERT Marina, 2012, Kooperative und unkooperative Verwendung von Pronomen in Texten der Physik und der Literatur (Kafka, Thomas Mann) aus dem frühen 20. Jahrhundert, in: Zeitschrift des Verbandes Polnischer Germanisten 1, S. 50-73, auch in: http://www.ejournals. eu/ZVPG/Tom-1(2012)/Zeszyt-1(2012)/art/1821/.

FosCHI AlBERT Marina, 2015, La negazione in poesia e l'uso "poetico" di nicht, in: Ballestracci S./ Grazzini S. (Hrsg.), Punti di vista - Punti di contatto. Studi di letteratura e linguistica tedesca, Firenze, S. 15-39, auch in: http://www.fupress.com/catalogo/punti-di-vista-\%E2\%80\%93-punti-di-contatto--studi-di-letteratura-e-linguistica-tedesca/2913.

FosCHI AlBERT Marina, 2016, Ambiguität sprachlicher Mittel als poetische Qualität, in: Zhu J./ Zhao J./Szurawitzki M. (Hrsg.), Germanistik zwischen Tradition und Innovation. Akten des XIII. Kongresses der Internationalen Vereinigung für Germanistik (IVG), Shanghai, 23.-30.8. 2015. Frankfurt am Main u.a., S. 241-245.

GÉRARD Genette, 1992, Fiktion und Diktion, München.

GRICE Paul H., 1979, Logik und Konversation, in: Meggle G. (Hrsg.), Handlung, Kommunikation, Bedeutung, Frankfurt am Main, S. $243-265$.

Holt Nadine van / Groeben Norbert, 2005, Das Konzept des Foregrounding in der modernen Textverarbeitungspsychologie, in: Journal für Psychologie 13/4, S. 311-332.

JAKOBSON Roman, 1971 [1960], Linguistik und Poetik, in: Ihwe J. (Hrsg.), Literaturwissenschaft und Linguistik. Ergebnisse und Perspektiven, Frankfurt am Main, S. 142-178. 
JAKOBSON Roman, 1972, Über die neueste russische Poesie, in: Texte des russischen Formalismus, hrsg. von W.-D. Stempel, München, S. 31-33.

KAFKA Franz, 1994, Gesammelte Werke in zwölf Bänden. Nach der kritischen Ausgabe herausgegeben von Hans-Gerd Koch. Bd. 1. Ein Landarzt und andere Drucke zu Lebzeiten, Frankfurt am Main.

KLEEFELD Gunther, 1985, Das Gedicht als Sühne. Georg Trakls Dichtung und Krankheit. Eine psychoanalytische Studie, Tübingen.

KLEIN Wolfgang / WINKLER Susanne, 2010, Einleitung, in: LiLi. Zeitschrift für Literaturwissenschaft und Linguistik 40, 158 [Ambiguität. Hrsg. von Klein W./Winkler S.], S. 5-6.

KNAPE Joachim / SIEBER Armin, 1998, Rhetorik-Vokabular zur zweisprachigen Terminologie in älteren deutschen Rhetoriken, unter Mitarbeit von Geier Andrea, Wiesbaden.

LANG Ewald, 2010, Der Ton macht den Sinn. Prosodische Differenzierungen bei syntaktischer Indifferenz als Lehrstoff, in: LiLi. Zeitschrift für Literaturwissenschaft und Linguistik 40, 158 [Ambiguität. Hrsg. von Klein W./Winkler S.], S. 172 - 188.

LEISS Ingo / STADLER Hermann, 1997, Wege in die Moderne 1890 - 1918, München.

Miall David S., 1977, Metaphor and literary meaning, in: British Journal of Aesthetics17/1, S. $49-59$.

MUKAR̆OVSKÝ Jan, 1967 [1948], Kapitel aus der Poetik, Frankfurt am Main.

Poetik $=$ Aristoteles. Über Die Dichtkunst. Neu übersetzt und mit Einleitung und einem erklärenden Namen- und Sachverzeichnis versehen von Gudeman Alfred, 1921, auch in: <https://www. gutenberg.org/files/16880/16880-0.txt>.

PoteBnJA Aleksandr Afans'evic, 1862, Mysl' i iazyk (,Gedanke und Sprache'), in: Zhurnal' Ministerstva Narodnago Prosveshcheniia', Sanktpeterburg 113, II, S. $1-118$.

RINSUM Annemarie van / RINSUM Wolfgang van, 1995, Interpretationen. Lyrik, München.

SANDIG Barbara, ${ }^{2} 2006$, Textstilistik des Deutschen, Berlin/New York.

SCHROTT Raoul / JACOBS Arthur, 2011, Gehirn und Gedicht. Wie wir unsere Wirklichkeiten konstruieren, München.

SCHWARZE Christoph, 1970, Untersuchungen zum syntaktischen Stil der italienischen Dichtungssprache bei Dante, Bad Homburg v. d. H./Berlin/Zürich.

Tsur Reuven, ${ }^{2}$ 1992, Toward a Theory of Cognitive Poetics, Brighton/Portland.

UhMANN Susanne, 1991, Fokusphonologie, Tübingen.

WINKO Simone, 2009, Auf der Suche nach der Weltformel. Literarizität und Poetizität in der neueren literaturtheoretischen Diskussion, in: Winko S./Jannidis F./Lauer G. (Hrsg.), Grenzen der Literatur. Zum Begriff und Phänomen des Literarischen, Berlin/New York, S. 374-396.

Winter-Froemel Esme / ZirKel Angelika, 2010, Ambiguität in der Sprecher-Hörer-Interaktion. Linguistische und literaturwissenschaftliche Perspektive, in: LiLi. Zeitschrift für Literaturwissenschaft und Linguistik 40, 158 [Ambiguität. Hrsg. von Klein W./Winkler S.], S. 76-97.

Wolf Maryanne, 2007, Proust and the Squid: The Story and Science of the Reading Brain.

ZIEGLER René, 2010, Ambiguität und Ambivalenz in der Psychologie, in: LiLi. Zeitschrift für Literaturwissenschaft und Linguistik 40, 158 [Ambiguität. Hrsg. von Klein W./Winkler S.], S. $125-171$. 